\title{
Optimization of an Asymmetric DFB Laser Used as All-Optical Flip-Flop
}

\author{
Amin Abbasi, Student Member, IEEE, Gunther Roelkens, Member, IEEE, \\ and Geert Morthier, Senior Member, IEEE
}

\begin{abstract}
We have numerically optimized the speed of an all-optical flip-flop based on an antireflection-coated $\lambda / 4$ phaseshifted distributed feedback (DFB) laser. Numerical results show that by engineering the grating of the DFB laser and making the coupling coefficient asymmetrical, fall times reduce by a factor of 2 compared with a standard laser design. It is also shown that the confinement factor in the quantum wells and the length of the laser cavity have a significant impact on the flip-flop operation. The switching between two states can be realized using pulses with energies below $125 \mathrm{fJ}$ and with a duration of $10 \mathrm{ps}$. The switching time can be reduced to 5 ps and the switching rate increased up to $10 \mathrm{GHz}$.
\end{abstract}

Index Terms-All-optical flip-flops, distributed feedback (DFB) lasers, optical bistability.

\section{INTRODUCTION}

A LL-OPTICAL devices are expected to play an essential role in future optical data networks [1]. In order to increase the network efficiency and raise bandwidth, all-optical switching networks are desirable, because they are signal format and bitrate transparent and can reduce the switching energy by avoiding optical-to-electrical or electrical-to-optical signal conversion [2], [3]. In optical packet switching networks, optical memory elements are required for holding the decisions from the optical header processors and for providing the label signals to the optical switches. It is impossible though to mimic the existing electronic memories in the optical domain because of the electrical neutrality of photons. Nevertheless, some work has recently been presented on optical random access memories (RAM) [4], [5], which could open new directions in all-optical networking. A digital optical memory, implemented via an all-optical flip-flop (AOFF), can be used to store an optical bit with almost infinite duration [6]-[14].

Most of the AOFF structures that have been proposed so far are based on bistable laser diodes, e.g. micro-disk and microring lasers [6]-[8], multimode interference (MMI) bistable lasers [9], photonic crystal lasers [10] and polarization bistable vertical-cavity surface-emitting lasers (VCSELs) [11], [12]. Recently, these all-optical flip-flop structures have been employed for all-optical packet based routing [13].

Manuscript received September 12, 2014; revised October 29, 2014; accepted November 10, 2014. Date of publication December 9, 2014; date of current version December 16, 2014. This work was supported by the Methusalem Program through the Flemish Government.

The authors are with the Photonics Research Group, Center for Nano and Biophotonics, Ghent University-imec, Ghent 9000, Belgium (e-mail: aamin@intec.ugent.be; gunther.roelkens@intec.ugent.be; geert.morthier@intec.ugent.be).

Color versions of one or more of the figures in this paper are available online at http://ieeexplore.ieee.org.

Digital Object Identifier 10.1109/JQE.2014.2378376

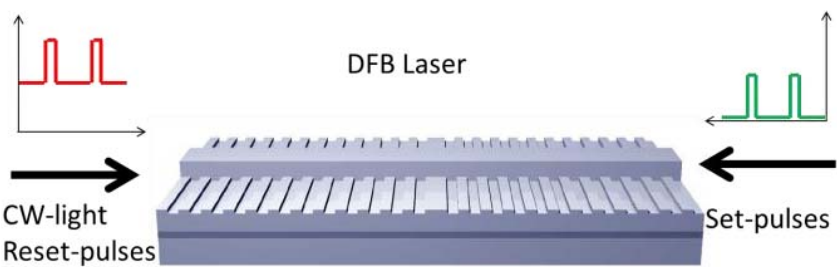

Fig. 1. Schematic representation of the all-optical flip-flop.

The AOFF based on an anti-reflection-coated (AR-coated) $\lambda / 4$-shifted distributed feedback (DFB) laser diode, which has been already experimentally demonstrated [14], [15], has the main advantage that it can operate over a broad wavelength range for the set and reset pulses and that it is based on mature device technology. It requires externally injected light, but there are no hard wavelength requirements for the external beam. The operation principle is outlined in Fig. 1. A CW beam is injected from the left hand side to have two stable states. This bistability is based on the non-uniform axial distribution of the carrier density (longitudinal spatial hole burning). By injecting a short but strong pulse at the same side of the device as the $\mathrm{CW}$ beam, one can switch from a lasing state (with low gain and uniform carrier density) to a non-lasing one (with high gain and non-uniform carrier density). The uniform carrier distribution can then be restored by injecting a light pulse at the other side of the device [15]. The influence of the bias current and normalized coupling coefficient $(\kappa \mathrm{L})$ on the bistability has been investigated before [14].

In this work, we propose a new design for the AOFF, which effectively improves the switching characteristics. By analyzing the impact of important parameters on the switching performance, the laser structure has been optimized for high speed operation. A high confinement factor in the active region and an asymmetric coupling coefficient (ACC) are the main factors that have a positive impact on the flip-flop operation. The cavity length and the carrier lifetime are also optimized to enable operation with short reset-set pulses. Our approach for the all-optical flip-flop switching is based on a $\lambda / 4$-shifted AR-coated DFB laser, which becomes bistable by injecting a holding beam (HB) from another laser, with a wavelength which is outside of the DFB laser stop-band. The bistability is due to non-linear effects having their origins in the carrier density distribution (i.e. longitudinal spatial hole burning).

\section{INFLUENCE OF THE CONFINEMENT FACTOR}

In a semiconductor laser, the confinement factor is defined as the ratio of the light intensity in the active region to 
TABLE I

DeVice Parameters USED in the Simulations

\begin{tabular}{ll}
\hline \hline Parameter & Value \\
\hline active region thickness $[\mu \mathrm{m}]$ & 0.040 \\
active region width $[\mu \mathrm{m}]$ & 2.5 \\
$\mathrm{SCH}$ layer thickness $[\mu \mathrm{m}]$ & 0.210 \\
facet reflectivity & $1 \mathrm{e}-12$ \\
differential gain coefficient $\left[\mathrm{m}^{2}\right]$ & $5 \mathrm{e}-20$ \\
gain saturation coefficient $\left[\mathrm{m}^{3}\right]$ & $1 \mathrm{e}-23$ \\
Linewidth enhancement factor & 3 \\
initial carrier density $\left[\mathrm{m}^{-3}\right]$ & $1 \mathrm{e} 24$ \\
linear recombination $\left[\mathrm{s}^{-1}\right]$ & 0 \\
bimolecular recombination $\left[\mathrm{m}^{3} / \mathrm{s}\right]$ & $1 \mathrm{e}-16$ \\
auger recombination $\left[\mathrm{m}^{6} / \mathrm{s}\right]$ & $1.3 \mathrm{e}-41$ \\
transparency carrier density $\left[\mathrm{m}^{-3}\right]$ & $1.5 \mathrm{e} 24$
\end{tabular}

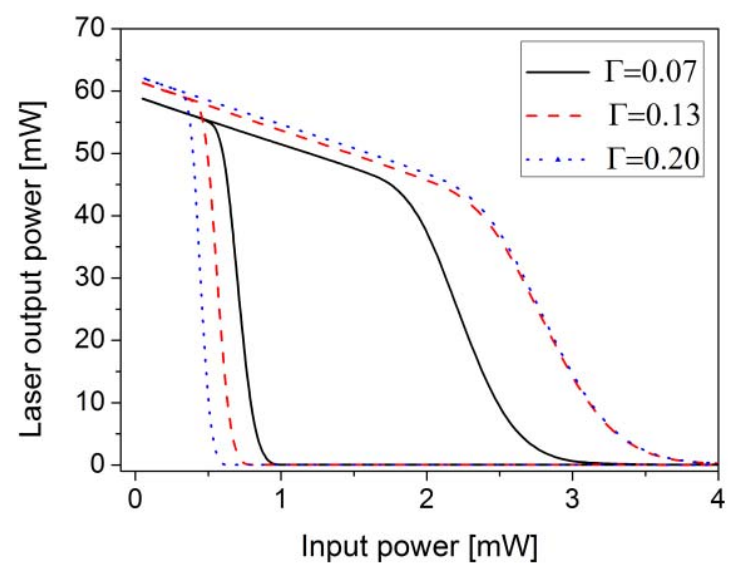

Fig. 2. Influence of the confinement factor on the hysteresis characteristics for a DFB laser of length $400 \mu \mathrm{m}$ and $\kappa \mathrm{L}=1.2$. The threshold currents corresponding with $\Gamma=0.07,0.13$ and 0.20 are $\mathrm{I}_{\mathrm{th}}=17.5 \mathrm{~mA}, 7 \mathrm{~mA}, 5 \mathrm{~mA}$ respectively and the laser was biased at $\mathrm{I}=240 \mathrm{~mA}$.

the total mode intensity. The confinement factor determines the carrier-photon interaction strength. Since this interaction plays a fundamental role for our all-optical flip-flop system, it will affect the flip-flop performance. We have investigated the influence of this parameter on the bistability and the switching dynamics (determined from the laser output power versus time). The dynamics of the AOFF are simulated with the commercial software package VPI [16]. The common parameters for all simulated DFB laser diodes are summarized in Table I.

In Fig. 2, the influence of the quantum well (QW) confinement factor on the bistability is shown. The laser length is $400 \mu \mathrm{m}$ and the DFB has a normalized coupling coefficient of 1.2. It is clear that an increase in the confinement factor not only results in a higher output power, but also widens the hysteresis loop. For a constant active layer volume, a higher confinement factor results in a lower threshold current for the laser. Hence, more input power is required to switch the laser off when the laser is initially lasing. In the off state, when the laser is not lasing, a higher confinement results in a higher stimulated emission rate and gain for the injected holding beam and hence more spatial hole burning. For a certain optical input power, this translates into a higher loss for the laser mode. Since the laser will switch back on when the loss
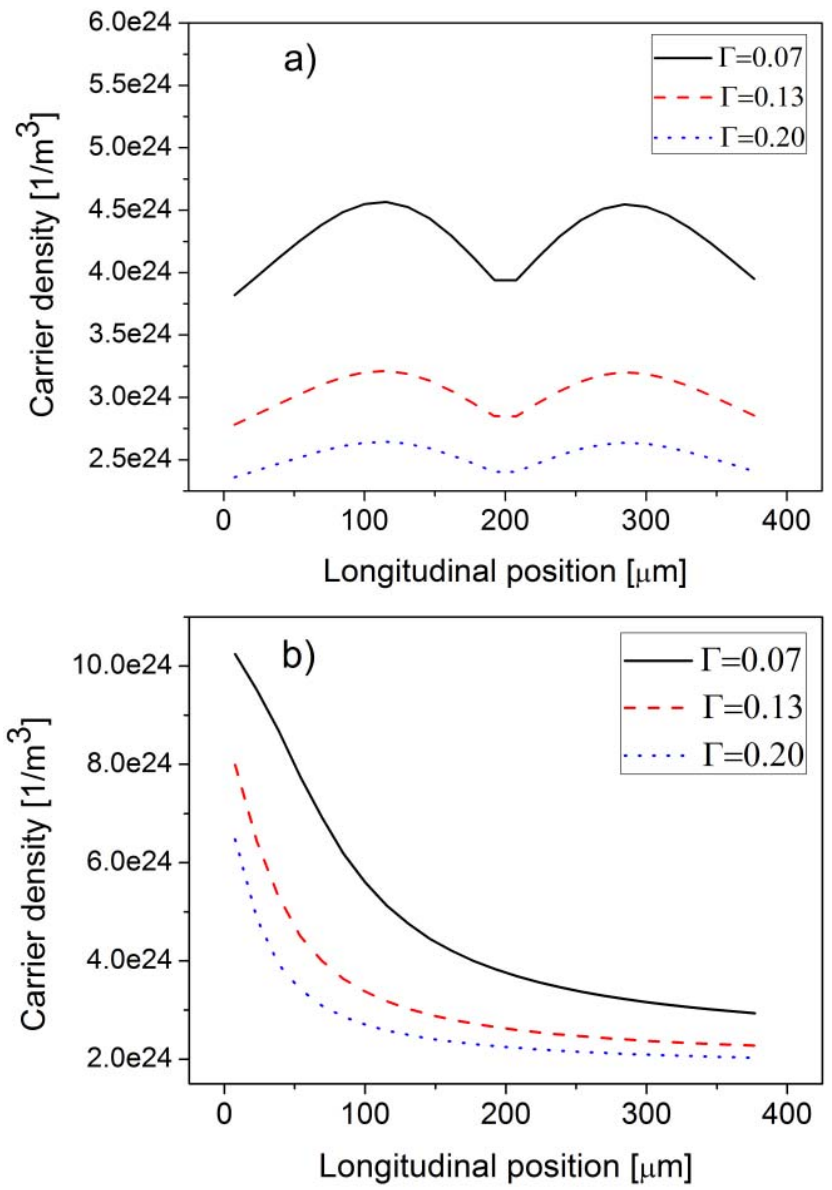

Fig. 3. Influence of the confinement factor on the longitudinal carrier density distribution when the laser is on (a) and off (b) for a DFB laser of length $400 \mu \mathrm{m}, \kappa \mathrm{L}=1.2$, and $\mathrm{I}=240 \mathrm{~mA}$. The left and right hand sides are corresponding to the rear and front facet, respectively.

has decreased sufficiently, and since the loss decreases with decreasing input optical power, a laser with higher confinement factor will switch back on for lower input power than a laser with lower confinement factor.

In Fig. 3(a) and (b) the longitudinal carrier density distributions are shown for the two states of the switching. The slope of the curve in the off state for the higher confinement factor is high in the first $100 \mu \mathrm{m}$ and then it levels off in the remaining laser length.

The modal gain can be calculated along the laser length using the formula

$$
\Gamma g=\Gamma a\left(N-N_{t r}\right)
$$

where $g$ is the gain, $N_{t r}$ is the transparency carrier density and $a$ is the differential gain coefficient. From the calculations, it follows that the confinement factor has little to no influence on the gain in the middle and right hand side (front facet) part of the cavity. While on the left hand side (rear facet) where strong non-uniformity is created (Fig. 3(b)), the modal gain rises with increasing confinement factor (e.g., at the extreme left, it increases from $300 \mathrm{~cm}^{-1}$ to $420 \mathrm{~cm}^{-1}$ for $\Gamma=0.07$ and $\Gamma=0.13$ respectively). Consequently, set pulses will experience the higher modal gain where they are supposed to re-establish the carrier density uniformity. 

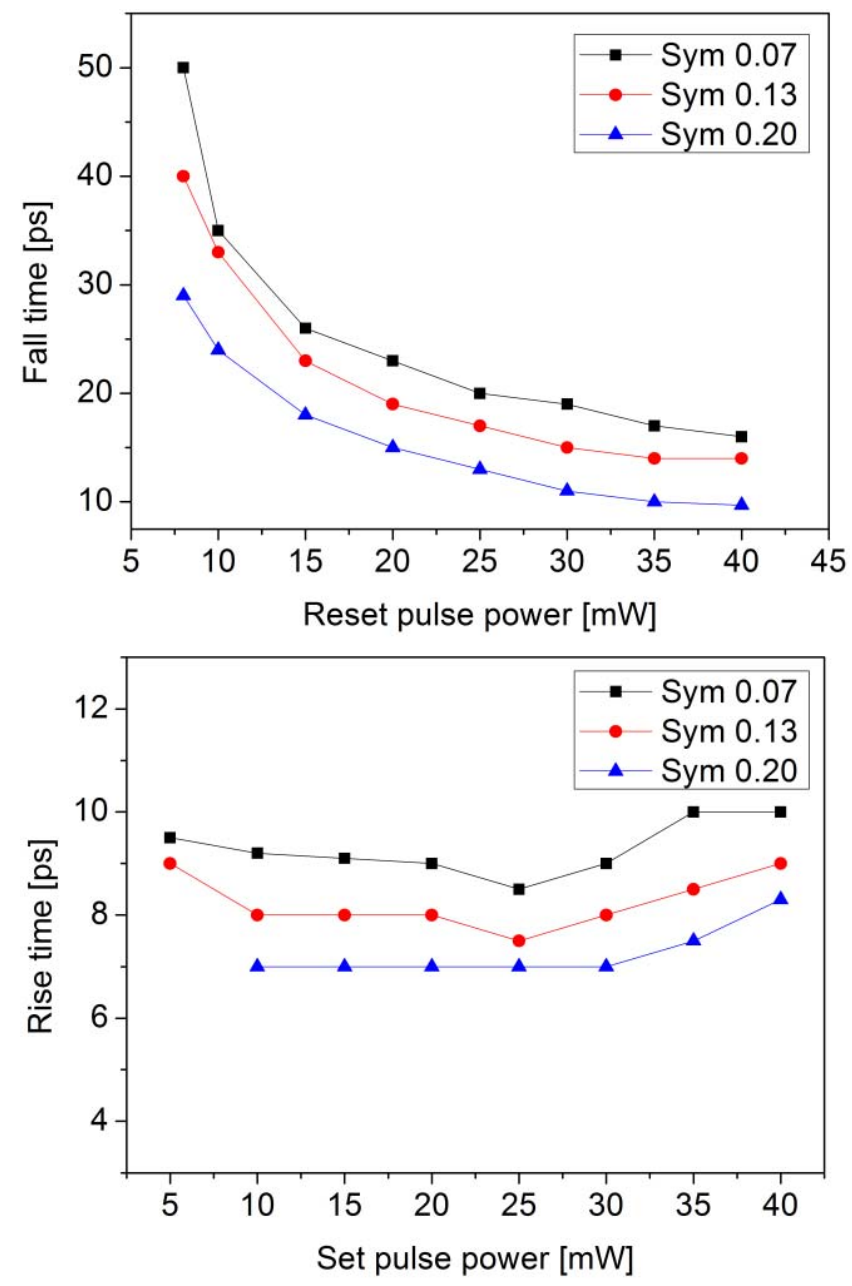

Fig. 4. The fall time (top) and rise time (bottom) for a DFB laser with length $\mathrm{L}=400 \mu \mathrm{m}, \kappa \mathrm{L}=1.2$ and $\mathrm{I}=240 \mathrm{~mA}$, for a quantum well confinement factor of $0.07,0.13$ and 0.20 respectively. The rectangular pulses have a duration of $100 \mathrm{ps}$ for both reset and set.

As already demonstrated, one can exploit this bistability for all-optical flip-flop operation. Besides the holding beam which provides bistability, pulses are injected at both sides of the laser diode to change the state of the system between the on and off. The required duration of the switching pulses can vary from $200 \mathrm{ps}$ to $10 \mathrm{ps}$ depending on the laser parameters. For instance, for a laser with lower confinement factor or symmetric grating structure switching is not possible using short pulses.

In Fig. 4 the reset and set pulse durations are kept at 100 ps. By increasing the reset pulse power, the fall time reduces accordingly. Improvement in the switching time by increasing the confinement factor is clear. Fall and rise times as low as $10 \mathrm{ps}$ and 7 ps can be achieved. The symbol "sym" on the figures refers to a $\lambda / 4$-shifted AR-coated DFB laser with a symmetric coupling coefficient.

\section{INFLUENCE OF THE ASYMMETRIC COUPLING COEFFICIENT}

In a DFB laser, an asymmetric coupling coefficient (ACC) can be used to increase the front facet output power as well as the single mode stability at high drive current [17].
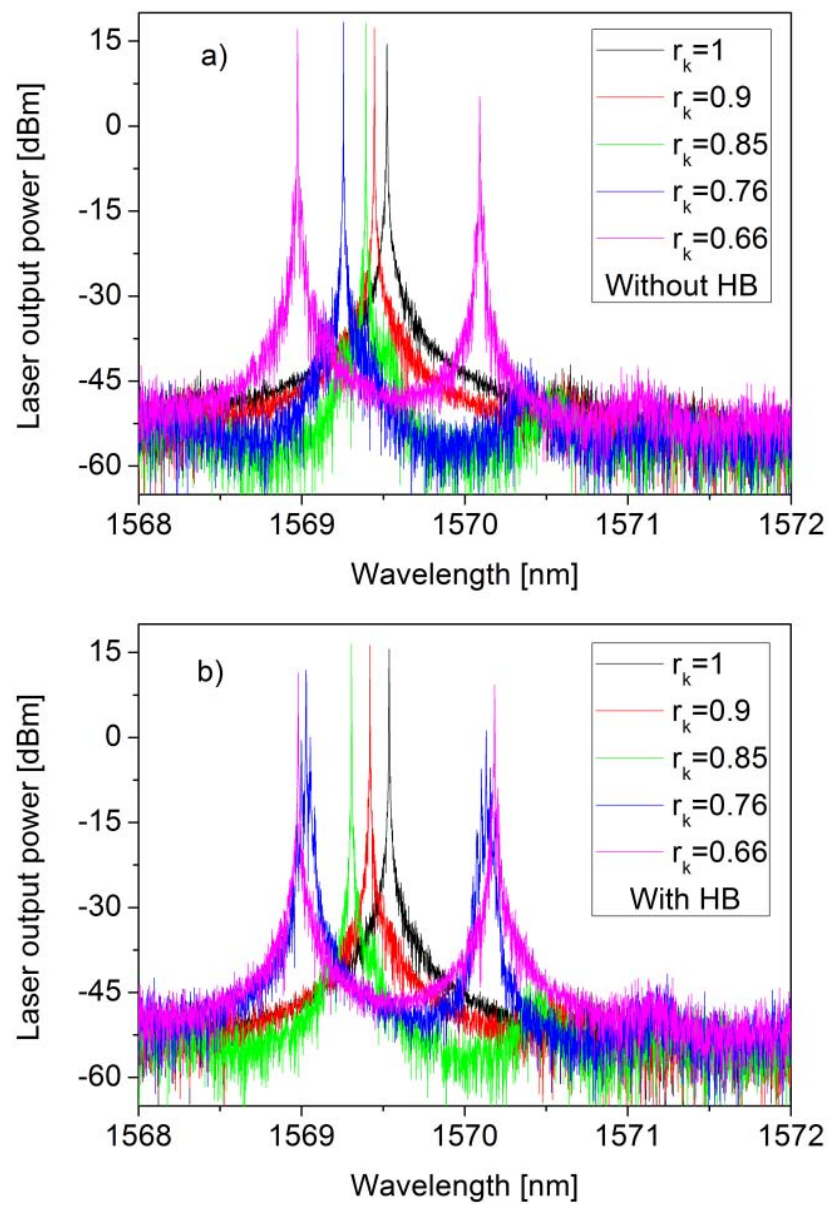

Fig. 5. The DFB laser spectrum for various values of $r_{\kappa}$ a) without b) with the holding beam at the injection current of $240 \mathrm{~mA}$. $\mathrm{L}=400 \mu \mathrm{m}$ and $\Gamma=0.13$.

In this section we will present a DFB laser with ACC in order to enhance the all-optical switching properties. The asymmetry is expressed by $r_{\kappa}$, which is defined as the ratio of the coupling coefficient on the right hand side of the phase shift to the coupling coefficient on the left hand side of it. The position of the phase shift is always in the middle of the laser cavity, only the value of the coupling coefficients on either side of the phase shift is different.

From a fabrication point of view, the asymmetry can be easily realized by changing the duty cycle of the grating slightly. The holding beam will be injected from the left hand side where the coupling coefficient is higher. This will increase longitudinal spatial hole burning (LSHB) while the laser is on. For the DFB laser without the holding beam, the laser can be stable and single mode even for a relatively strong asymmetry $\left(r_{\kappa}=0.76\right)$ (Fig. 5-a).When we are applying the holding beam, which increases the LSHB, the single mode operation of the laser deteriorates easily (Fig. 5-b).

The bistability curve for a symmetric and asymmetric DFB structure is shown in Fig. 6. Because of the stronger LSHB in the asymmetric case, the bistability threshold is moved toward lower input power. At the same time, the laser output is increased from the front facet (the right hand side) due to the lower coupling coefficient on the right hand side. On the other hand, the total coupling coefficient in the 


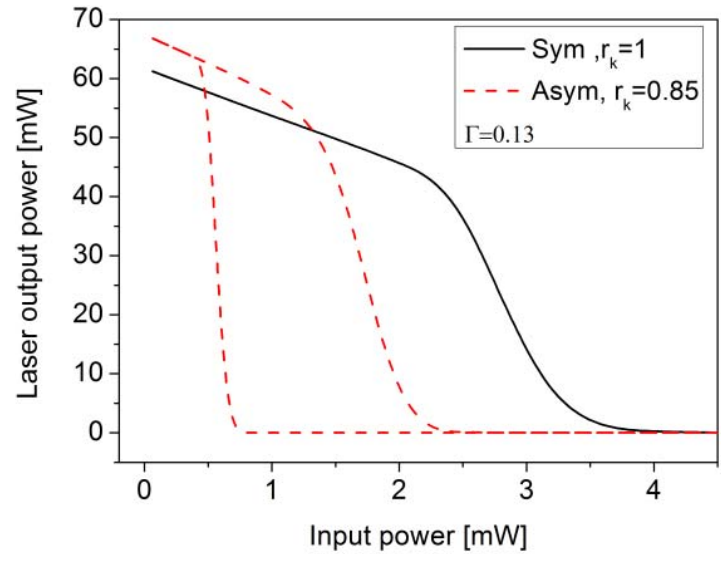

Fig. 6. The bistability for the symmetric and asymmetric coupling $\left(\mathrm{r}_{\kappa}=0.85\right)$ coefficient with $\mathrm{L}=400 \mu \mathrm{m},(\kappa \mathrm{L})_{\text {sym }}=1.2,(\kappa \mathrm{L})_{\text {asym }}=1.1$, $\Gamma=0.13$ and $\mathrm{I}=240 \mathrm{~mA}$.
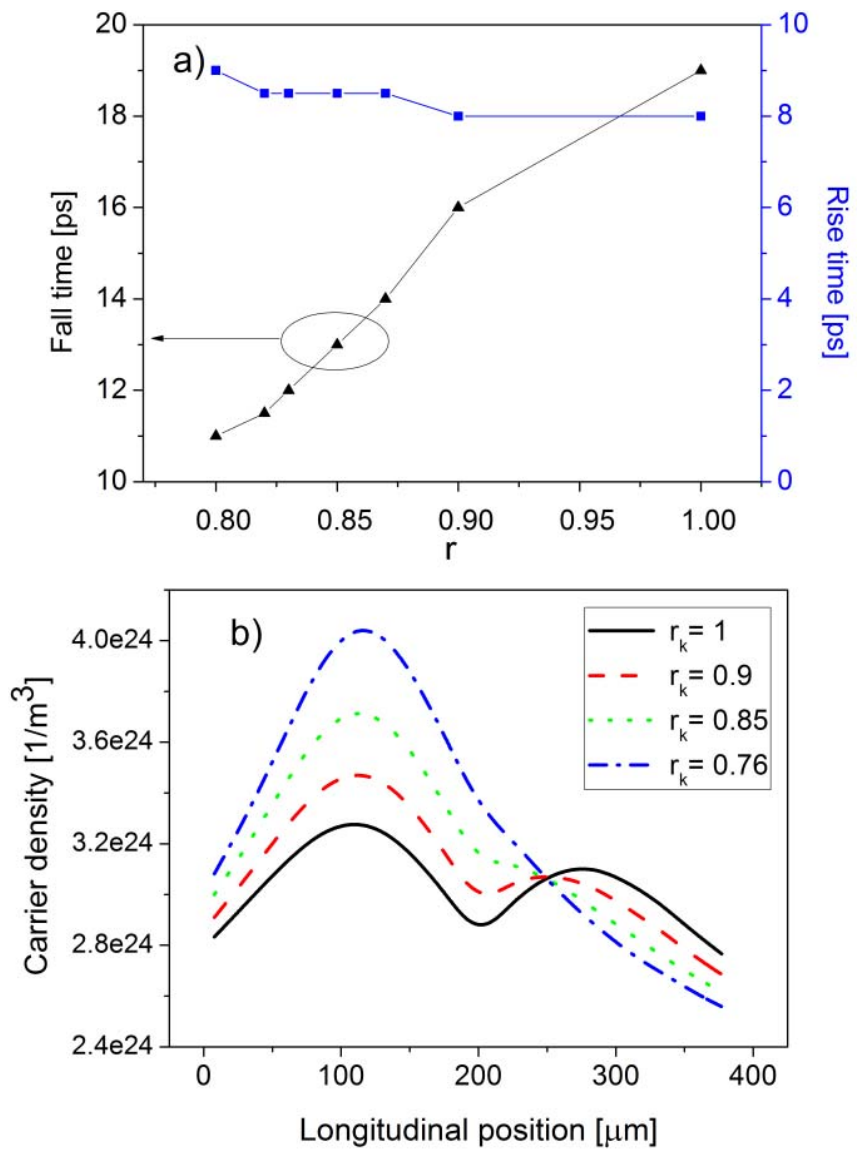

Fig. 7. The switching time (a) and the longitudinal carrier density distribution in the on-state (b) versus the asymmetry coefficient. $\mathrm{L}=400 \mu \mathrm{m}$ and $\mathrm{I}=240 \mathrm{~mA}$.

asymmetric laser is lower than the value for the symmetric one, which causes an increase in the threshold current of the asymmetric laser. This fact shrinks the bistability region, which can be compensated by increasing the injected current.

The dynamical properties of the asymmetric configuration were simulated for different asymmetry coefficients $r_{k}$. The results are shown in Fig. 7(a). As expected, the fall time is reduced by increasing the asymmetry coefficient $\left(\mathrm{r}_{\mathrm{k}}\right)$. However, a further increase will make the laser unstable.
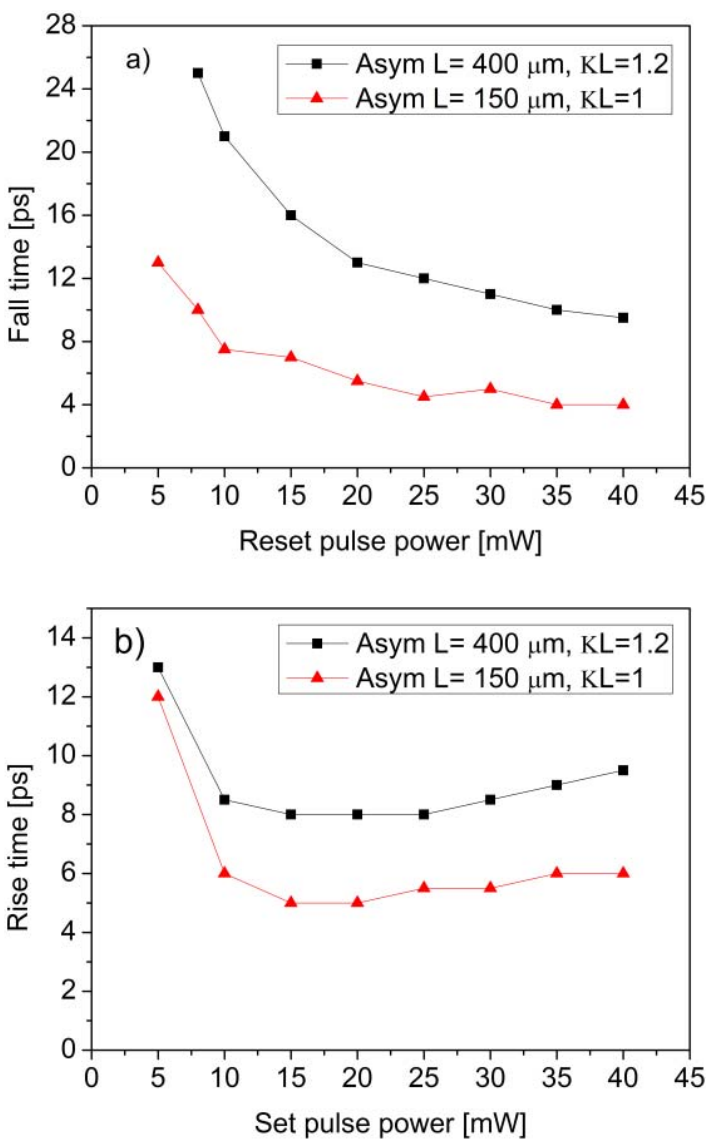

Fig. 8. The fall time (a) and rise time (b) for a DFB laser with length $\mathrm{L}=400 \mu \mathrm{m}, \kappa \mathrm{L}=1.1$ and $\mathrm{L}=150 \mu \mathrm{m}, \kappa \mathrm{L}=0.95$. The asymmetry coefficient $\left(\mathrm{r}_{\kappa}\right)$ is 0.85 and the drive current is $240 \mathrm{~mA}$ for both structures.

One can see that there is almost no effect on the rise time. Both reset and set pulses have a duration of $100 \mathrm{ps}$ and a power of $20 \mathrm{~mW}$.

In Fig. 7(b), the carrier density distribution when the laser is on as a function of asymmetry is shown, illustrating the stronger LSHB for a larger asymmetry coefficient.

\section{INFLUENCE OF THE LASER LENGTH}

As can be seen from the carrier density profiles in Fig. 3(b), for the high confinement factor even with a shorter cavity one can see strong longitudinal carrier non-uniformity. This was the motivation to investigate the dynamical properties for shorter cavities but with higher coupling coefficients so that the normalized coupling coefficients are kept close to unity.

In Fig. 8, the fall time and rise time for a laser with a length of $150 \mu \mathrm{m}$ are compared with those for a laser with a length of $400 \mu \mathrm{m}$. To keep the normalized coupling coefficient close to 1 , the coupling coefficients for the lengths of $400 \mu \mathrm{m}$ and $150 \mu \mathrm{m}$ are $30 \mathrm{~cm}^{-1}$ and $68 \mathrm{~cm}^{-1}$ respectively. $\mathrm{r}_{\kappa}$ equals 0.85 in both cases. The set and reset pulse widths are 25 ps.

For the optimized conditions and the asymmetric configuration, the fall time drops to very small values (e.g. for a pulse energy of $500 \mathrm{fJ}$ it is $5 \mathrm{ps}$ ). Switching for this structure is even possible with pulses with very low energy (125 fJ). 


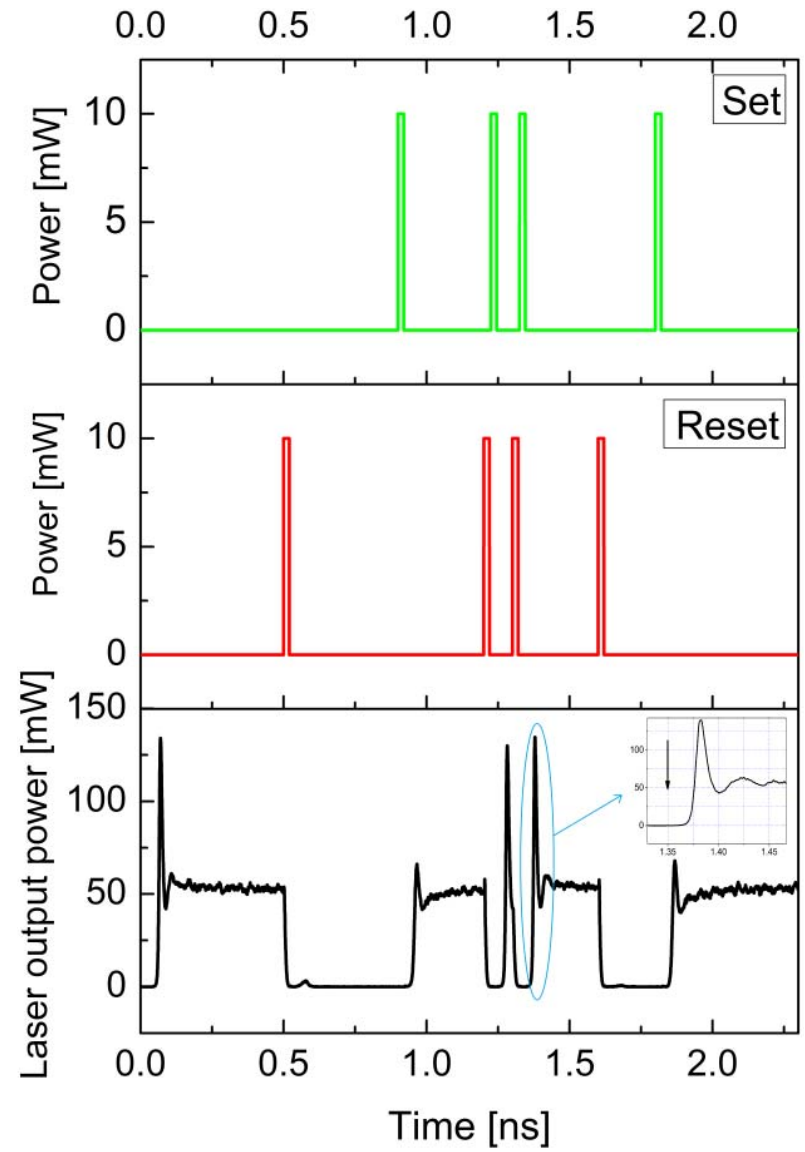

Fig. 9. Laser output waveform with random switching events. The holding beam power is $1.6 \mathrm{~mW}$ and pulse energies are $300 \mathrm{fJ} . \Gamma$ is $0.13, \mathrm{~L}=150 \mu \mathrm{m}$, $\kappa \mathrm{L} \approx 1$ and the asymmetry coefficient is $\mathrm{r}_{\kappa}=0.85, \mathrm{I}_{\mathrm{th}}=11 \mathrm{~mA}$ $\mathrm{I}=240 \mathrm{~mA}$.

The rise time can be half of that of the asymmetric long DFB laser. If we compare the results with the conventional long symmetric laser, the optimized design can switch much faster and with lower energy pulses. Switching times as low as 5 ps were observed in simulations with pulses of just $10 \mathrm{ps}$. A shorter cavity may suffer from increased heating, which can be solved by using a thermal shunt to the laser's metal contacts.

We were also able to obtain switching rates of $10 \mathrm{GHz}$ applying reset-set pulses with an energy of just $300 \mathrm{fJ}$ and a duration of 15 ps (Fig. 9).

As can be seen in the inset of Fig. 9, there is a delay time after applying the set pulse. This delay time, as well as a strong relaxation oscillation after the rise time limit the switching repetition rate. We found that if the drive current decreases to $150 \mathrm{~mA}$, switching at the same rate could still be achieved, although at the expense of a lower extinction ratio and a narrow bistable region. Under this condition, almost $30 \%$ reduction was obtained for the required holding beam power as well as for the switching pulse power.

\section{CONCLUSION}

We have carefully numerically investigated the effects of confinement factor, grating asymmetry and laser cavity length on the operation of a $\lambda / 4$-shifted DFB laser all-optical flipflop. This has led to some clear and simple design guidelines for the optimization of the DFB laser structure, such that it can be operated at high switching rate, with very short switching times and low switching energy.

Practical applications of the device could be in optical (RAM) memories for WDM networks, since the wavelength of the holding beam and switching pulses is not limited to a pre-defined value [18], [19], which makes the device very flexible.

\section{REFERENCES}

[1] G. P. Agrawal, Lightwave Technology: Telecommunication Systems. New York, NY, USA: Wiley, 2005.

[2] P. R. Prucnal, V. Baby, D. Rand, B. C. Wang, and I. Glesk, "All-optical processing in switching networks," IEEE LEOS Newslett., vol. 16, no. 4 pp. 13-14, Oct. 2002.

[3] H. J. S. Dorren et al., "Optical packet switching and buffering by using all-optical signal processing methods," J. Lightw. Technol., vol. 21, no. 1 , pp. 2-12, Jan. 2003.

[4] G. Berrettini, L. Poti, and A. Bogoni, "Optical dynamic RAM for all-optical digital processing," IEEE Photon. Technol. Lett., vol. 23, no. 11, pp. 685-687, Jun. 1, 2011.

[5] D. Fitsios, K. Vyrsokinos, A. Miliou, and N. Pleros, "Memory speed analysis of optical RAM and optical flip-flop circuits based on coupled SOA-MZI gates," J. Sel. Topics Quantum Electron., vol. 18, no. 2, pp. 1006-1015, Mar./Apr. 2012.

[6] L. Liu et al., "An ultra-small, low-power, all-optical flip-flop memory on a silicon chip," Nature Photon., vol. 4, pp. 182-187, Jan. 2010.

[7] M. T. Hill et al., "A fast low-power optical memory based on coupled micro-ring lasers," Nature, vol. 432, pp. 206-209, Nov. 2004.

[8] A. Trita et al., "Dynamic operation of all-optical flip-flop based on a monolithic semiconductor ring laser," in Proc. 34th Eur. Conf. Opt. Commun., Sep. 2008, pp. 1-2, paper We2C3.

[9] M. Takenaka, M. Raburn, and Y. Nakano, "All-optical flip-flop multimode interference bistable laser diode," IEEE Photon. Technol. Lett., vol. 17, no. 5, pp. 968-970, May 2005.

[10] C.-H. Chen et al., "All-optical memory based on injection-locking bistability in photonic crystal lasers," Opt. Exp., vol. 19, no. 4, pp. 3387-3395, 2011.

[11] T. Mori, Y. Yamayoshi, and H. Kawaguchi, "Low-switching-energy and high-repetition-frequency all-optical flip-flop operations of a polarization bistable vertical-cavity surface-emitting laser," Appl. Phys. Lett., vol. 88, no. 10, pp. 101102-1-101102-3, Mar. 2006.

[12] T. Katayama, T. Kitazawa, and H. Kawaguchi, "All-optical flip-flop operation using $1.55 \mu \mathrm{m}$ polarization bistable VCSELs," in Proc. Conf. Lasers Electro-Opt./Quantum Electron. Laser Sci. Conf., May 2008, pp. 1-2, paper CME5.

[13] C. Reis et al., "Experimental analysis of an all-optical packet router," IEEE/OSA J. Opt. Commun. Netw., vol. 6, no. 7, pp. 629-634, Jul. 2014.

[14] K. Huybrechts, W. D'Oosterlinck, G. Morthier, and R. Baets, "Proposal for an all-optical flip-flop using a single distributed feedback laser diode," IEEE Photon. Technol. Lett., vol. 20, no. 1, pp. 18-20, Jan. 1, 2008.

[15] K. Huybrechts, G. Morthier, and R. Baets, "Fast all-optical flip-flop based on a single distributed feedback laser diode," Opt. Exp., vol. 16, no. 15, pp. 11405-11410, 2008.

[16] VPI transmission Maker. [Online]. Available: http://www.vpisystems. com

[17] O. K. Kwon, Y. A. Leem, D. H. Lee, C. W. Lee, Y. S. Baek, and Y. C. Chung, "Effects of asymmetric grating structures on output efficiency and single longitudinal mode operation in $\lambda / 4$-shifted DFB laser," IEEE J. Quantum Electron., vol. 47, no. 9, pp. 1185-1194, Sep. 2011.

[18] G. T. Kanellos, D. Fitsios, T. Alexoudi, C. Vagionas, A. Miliou, and N. Pleros, "Bringing WDM into optical static RAM architectures," J. Lightw. Technol., vol. 31, no. 6, pp. 988-995, Mar. 15, 2012.

[19] E. Kuramochi et al., "Large-scale integration of wavelength-addressable all-optical memories on a photonic crystal chip," Nature Photon., vol. 8, pp. 474-481, May 2014. 


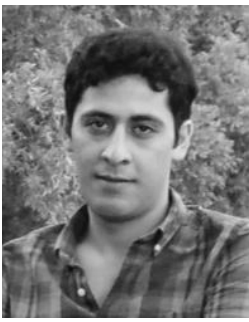

Amin Abbasi received the B.Sc. degree in applied physics and the M.Sc. degree in laser physics from the University of Tabriz, Tabriz, Iran, in 2007 and 2009, respectively. He is currently pursuing the Ph.D. degree in photonics with the Photonics Research Group, Department of Information Technology, Ghent University-imec, Ghent, Belgium.

His current research interests include heterogeneously integration of silicon-on-isolator and III-V materials and all-optical signal processing based on distributed feedback (DFB) and distributed Bragg reflector (DBR) lasers.

Mr. Abbasi is a Student Member of the IEEE Photonics Society.

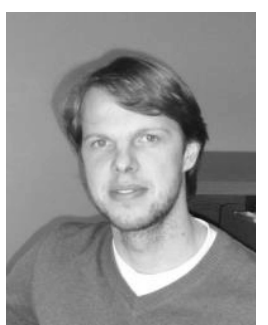

Gunther Roelkens received the degree in electrical engineering from Ghent University-imec, Ghent, Belgium, in 2002, and the Ph.D. degree from the Department of Information Technology, Ghent University-imec, in 2007, where he is currently a Research Professor. In 2008, he was a Visiting Scientist with the IBM Thomas J. Watson Research Center, Yorktown Heights, NY, USA. He is also a part-time Professor with the Eindhoven University of Technology, Eindhoven, The Netherlands. His research interests include the heterogeneous integration of III-V semiconductors and other materials on the top of silicon waveguide circuits, for high-performance photonic integrated circuits, both in the near and mid-infrared. He holds an ERC starting grant (MIRACLE), to start up research in the field of integrated mid-infrared photonic integrated circuits. He has authored over 100 journal papers. He is a member of the IEEE Photonics Society.

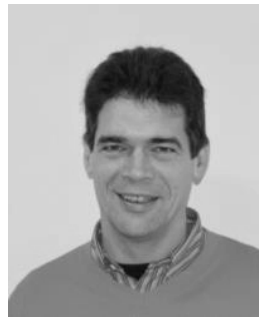

Geert Morthier (M'93-SM'01) received the degree in electrical engineering and the Ph.D. degree from Ghent University, Ghent, Belgium, in 1987 and 1991, respectively. Since 1991, he has been a member of the permanent staff with imec, Belgium. His main interests are in the modeling and characterization of optoelectronic components. He has authored or co-authored over 150 papers and holds several patents. He is also one of the two authors of the book entitled Handbook of Distributed Feedback Laser (Artech House, 1997 and 2013). From 1998 to 1999, he was the Project Manager of the ACTS project ACTUAL dealing with the control of widely tunable laser diodes, the IST project NEWTON on new widely tunable lasers from 2001 to 2005 , and the FP7 project HISTORIC on microdisk lasers from 2008 to 2011. In 2001, he was appointed as a parttime Professor at Ghent University, where he teaches courses on optical fiber communication and lasers.

He serves on the Program Committee of several international conferences. 